\title{
A Review: Techniques of Vehicle Detection in Fog
}

\author{
Rahul Singh*, Someet Singh and Navjot Kaur \\ Lovely Professonal University, Phagwara - 144411,Punjab, India: rkant225@gmail.com, someetsingh84@gmail.com, \\ dhhami.navjot@gmail.com
}

\begin{abstract}
Objective: Here we are going to describe the technique to detect vehicle in foggy environment. Vehicle detection in foggy weather is important because poor visibility is the major reason of the accidents and collision of vehicle. LiDAR and cameras are often used for better performance. Method: The image which is received from the camera in foggy condition is totally distorted and blurred and it will not clear up to the desired level so that the vehicle in front is clearly visible to us, so in order to deblur our image and make it clear we will use Adaptive Gaussian Thresholding Technique. In this technique threshold value is the weighted sum of the neighborhood pixel values which will make our image clearer and clean as compared to the original image. In addition with camera we are using low cost LiDAR which consists of a laser and a camera both of these devices are combined to measure accurate distance up to 10 meter. This LiDAR will used to measure the distance from front vehicle and provide warning according to the measured distance. Finding: The coding of this system is completely based on the python which is faster and lightweight as compared to the MATLAB. And our LiDAR is also more accurate and fast as compared to the traditional LiDAR system its major plus point is that it is of low cost. The combination of LiDAR and camera make our system more powerful and efficient.
\end{abstract}

Keywords: Adaptive Gaussian Thresholding, Computer Vision, LiDAR, Python

\section{Introduction}

As indicated by a study more than 10 years (2002-2012), just three percent $(3 \%)$ of climate related incidents happened because of fog. Despite the fact that incident created by this kind of perceivability issue speak to a little rate of aggregate mishaps on interstates, they are for the most part calamitous and savage. One of the tests confronted by vehicle vision applications is the effect of unfavorable conditions through sensors weakness, particularly cameras. Streamlining ADAS execution by moderating the impacts of climate on the roadways requires exact, convenient and solid data on current climate and perceivability conditions. Haze location and observing is however regularly inclined to the flighty way of mist with sudden changes and neighborhood varieties. Vehicle recognition is a point that has picked up significance recently as independent vehicles have picked up footing. In order to guarantee that self-sufficient vehicles can securely drive in situations with people in them, the vehicle must have the capacity to recognize and maintain a strategic distance from vehicle in short and long ranges, during day and night, keeping in mind in movement. Contingent upon the vehicle's speed, identifications as far out as $\mathbf{5 0}$ meters and past might be fundamental. As of late, various research ventures have been done in creating Driver Assist Systems (DAS). The reason for a DAS is to watch nature out and about and help drivers get movement data with high precision and effectiveness. A DAS can likewise be stretched out to robotized driving undertakings. One of the key undertakings of a DAS is identifying and following the encompassing autos around the DAS-prepared vehicle. Regarding sensors, one of the more mainstream detecting strategies is utilizing PC vision framework.

\section{Vehicle Detection System}

The vehicle detection framework is almost intended to give better wellbeing and make the vehicle self-governing. This framework is utilizing a Camera and a LiDAR for recognition of vehicle. These sensors recognize the

${ }^{*}$ Author for correspondence 
vehicle, protest, passerby and so forth and give data to the principle control unit and it settles on the choice as per it and controls the vehicle and give the notice flag to the driver.

\subsection{LiDAR}

LiDAR utilizes laser light to quantify separations. It is utilized as a part of numerous courses, from assessing climatic condition, shooting a laser bar to getting speeders in road activity with a handheld laser-speed locator. There are some laser-checking innovation which are spent significant time in various field for instance flying machine based sort of LiDAR that gives to a great degree precise, nitty gritty 3-D estimations of the ground, vegetation and structures. In open, level territories, ground shapes can be recorded from an airplane flying overhead giving exactness inside 6 inches of real height. In steep, forested zones precision is regularly in the scope of 1 to 2 feet and relies on upon numerous components, including thickness of shade cover and the separating of laser shots. The speed and exactness of LiDAR made it attainable to guide huge regions with the sort of detail that before had just been conceivable with tedious and costly ground review crews, LiDAR basically take a shot at the principal of speed and distance:

Distance $=($ speed of light $*$ Time taken $) / 2$.

\subsection{Camera}

A camera is a non-contact gadget that recognizes the light falling on its lenses and converts it into an electronic signal, which is then handled to deliver a picture or a video screen and perform diverse calculation. Camera will take picture from moving vehicle and it will send that picture to the central handling unit utilized as a part of this system. If the processor will find that the picture is not clear because of the mist then it will apply some calculation to make it clear. Basically camera is significant segment of our fog debluring framework.

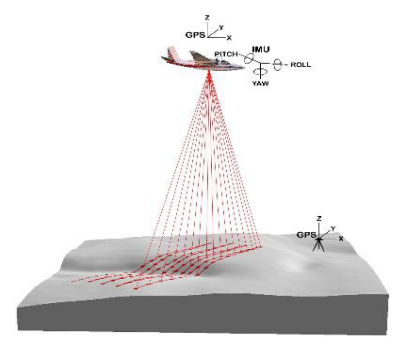

Figure 1. LiDAR projection on land from air $\mathrm{craft}^{\underline{8}}$.

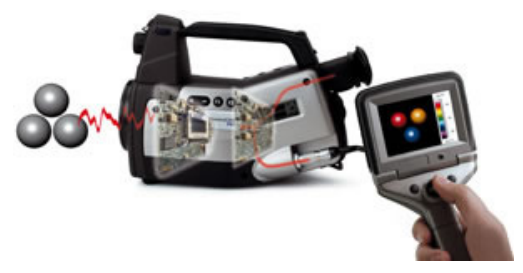

Figure 2. Camera ${ }^{9}$.

\section{Literature View 9}

Results of an extensive literature research in the field of Autonomous Driving System enhancement with more emphasis on the use of Vehicle Detection System are presented. The detailed work undertaken by various researchers and architects in the field of vehicle mechanization is displayed. The review incorporates hypothetical and in addition the test work attempted now and again alongside the advancement of the Vehicle Detection System for well being and security of human life.

The researcher Zsolt Kira has utilized numerous sensors to enhance the identification rate of passerby. Sensors utilized are LiDAR and IR camera. The contribution from various sensors is sent to course of classifiers, first depends on 'quick convolution organize classifier' and second is a 'HOG classifier'. These classifiers take choice in view of the information gave by the sensor and further control the diverse actuators, for example, caution, signal, streak light and so forth. At long last they have tried their framework on a Toyota Highlander vehicle. Both the Stationary and Dynamic (40 Kmph) conditions were tested ${ }^{1}$.

In next paper researchers have utilized Camera and LiDAR for discovery of vehicle. This paper shows a sensor combination based vehicle location approach by melding data from LiDAR and camera. The proposed approach depends on two segments, 1. Hypothesis era and 2. Hypothesis confirmation. Speculation era stage is to produce position that speak to vehicles and Hypothesis check stage is to arrange the comparing object. As confirmation researchers have utilized a SVM preparing process. Specialists gave 5000 preparing pictures with 1893 protests, pictures are physically named to prepare the classifier and 2000 test pictures for the assessment. Achievement rate for vehicle and non-vehicle grouping was $91 \%$.

In this paper Researchers have just utilized LiDAR to identify road paths and vehicle. Analysts likewise demonstrate the coordination of LiDAR and GPS for high exact route. For the most part LiDAR utilizes 3 mirrors 


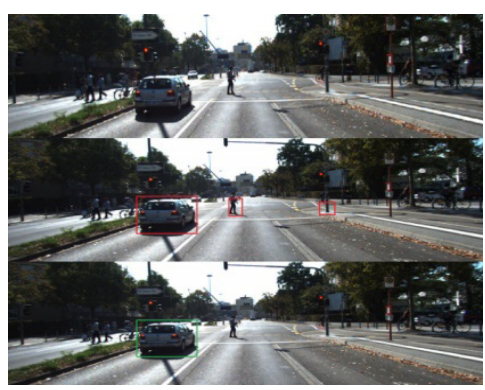

Figure 3. Classification result using HOG classifier $^{10}$.

to recognize the protest however Researchers utilized 6 reflect to identify all forward question including the path check. Discovery calculation is separated into three stages: First Researchers perceived the forward protest then they perceive the path check lastly road environment is perceived by incorporating these two sections. To discover the consequence of their framework analysts contrasted distinctive pictures got by framework and unique picture and inferred that the LiDAR can recognize different questions all the more precisely due to its day and night light emanation capability ${ }^{3}$.

In this paper researchers have utilized Camera and LiDAR to secure information about climate all around the vehicle. Researchers have talked about how information is gathered, broke down and converged to help the arrangement of moving vehicle in settling on self-ruling choice. Researchers began with taking Fourier change of the picture which contains the data about frequencies in the picture. At that point highlights from the picture are extricated utilizing the Gabor testing and Principal part Analysis (PCA). At that point these elements are encouraged to the classifier (SVM with RBF piece utilized) which has been prepared on mist and mist free pictures. Utilizing this technique they got $96 \%$ exactness in recognition of climate utilizing this system

In this paper, researchers have presented an estimation structure of various perceivability separations utilizing onboard CCD cameras, such as: Meteorological visibility, obstacle visibility, mobilized visibility. The strategies to appraise these distinctive visibility distances are clarified in this paper. Researchers have utilized Koschmieder's law to decide the mist impacts on climatic visibility ${ }^{5}$.

In this paper of researchers on the Driver Assistance System (DAS) is exhibited. They have utilized LiDAR and Computer vision sensor to make their framework more intelligent. Data discharged from both the LiDAR and camera are melded and sustained to classifier to recognize question and take choice. Ad boost classifier is utilized to group the protest, vehicle, pedestrian and so on. Their test comes about have demonstrated that when contrasted and picture based and exemplary sensor combination based vehicle recognition frameworks. This framework is very helpful for displaying and expectation of the movement conditions over an assortment of roadways. In this way it might be utilized as a part of future self-ruling route systems $s^{6}$.

This paper presents technique to use 2D LiDAR for route system to accomplish and enhance the $3 \mathrm{D}$ vehicle position estimation exactness. In this paper, 2D LiDAR estimation is done without forcing any presumptions on the vehicle movement. To pick up this element, a shut shape numerical expression is inferred to foresee the line estimation in the LiDAR's edge. This helps the element affiliation, GUI show and remaining development conceivable. With this expression, the Extended Kalman Filter can be actualized direct to consolidate the LiDAR and IMU information to evaluate the full condition of the vehicle. Last test comes about demonstrate the viability of the LiDAR supporting in diminishing the state estimation vulnerability in various directions ${ }^{\underline{7}}$.

\section{Conclusion}

It is a great challenge to detect vehicles under various foggy climatic scenarios. Surrounding objects such as pedestrians, trucks, trees and bicycles distract the system and cause the results to deviate from the actual scenario. LiDAR are often used to detect objects and find distance, whereas a vision based detection is responsible for real object verification and final classification. As compared with others, the benefit of this approach is to utilize the structural information to help vision based techniques for vehicle detection and classification. In this approach we are using low cost LiDAR model which is made up of the combination of a camera and a laser. It is accurate and of low cost as compared to the LiDAR.

\section{Future Scope}

Here we are working on 2D model, all the images collected and utilized are based on 2 dimensional approach. In future work we will concentrate on modeling the contour parameters from 2D models to 3D models. 


\section{References}

1. Zhang F, Clarke D, Knoll A. Vehicle detection based on LiDAR and camera fusion.17th International IEEE Conference on Intelligent Transportation Systems (ITSC); 2014. p. 1620-5.

2. Takagi K, Morikawa K, Ogawa T, Saburi M. Road environment recognition using on-vehicle LiDAR. 2006 IEEE Intelligent Vehicles Symposium; 2006. p. 120-5.

3. Dannheim C, Icking C, Mader M, Sallis P. Weather detection in vehicles by means of camera and LiDAR system. Sixth International Conference on Computational Intelligence, Communication Systems and Networks (CICSyN); 2014. p. $186-91$.

4. Hautiere N, Labayrade R, Aubert D. Detection of visibility conditions through use of onboard cameras. IEEE Proceedings of Intelligent Vehicles Symposium; 2005. p. 193-8.

5. Huang L, Barth M. Tightly-coupled LiDAR and computer vision integration for vehicle detection. 2009 IEEE Intelligent Vehicles Symposium; 2009. p. 604-9.
6. Zhao S, Farrell JA. 2D LiDAR aided INS for vehicle positioning in urban environment. IEEE International Conference on Control Applications (CCA); 2013. p. 376-81.

7. Lu G, Tomizuka M. LiDAR sensing for vehicle lateral guidance: Algorithm and experimental study. IEEE/ASME Transactions on Mechatronics. 2006; 11(6):653-60.

8. Image source available from: http://www.google. com/url? q=http $\% 3 \mathrm{~A} \% 2 \mathrm{~F} \% 2 \mathrm{Fforsys}$.cfr.washington. edu\%2FJFSP06\%2Flidar_technology.htm\&sa=D\&sntz=1\& usg=AFQjCNFBKr9KxhjRkSD4UF9MGG-FF-nZOQ

9. Image source available from: http://www.google.com/ url? q=http $\% 3$ A $\% 2$ F $\% 2$ Fwww.flir.com $\% 2$ Fcorporate $\% 2$ Fdisplay\%2F\%3Fid\%3D41523\&sa=D\&sntz=1\&usg =AFQjCNEmgdZozD6s01809VHEaskKyS92IA

10. Image source :

11. Zhang F, Clarke D, Knoll A. Vehicle detection based on LiDAR and camera fusion.17th International IEEE Conference on Intelligent Transportation Systems (ITSC); 2014. p. 1620-25. 